\title{
An Ultrasonic Interferometer for High-Pressure Research
}

\author{
Thomas J. Ahrens ${ }^{1}$ and Samuel Katz \\ Interdisciplinary Materials Research Center ${ }^{2}$ and Department of Geology \\ Rensselaer Polytechnic Institute, Troy, New York
}

\begin{abstract}
A new method in which ultrasonic interferometry is used to measure the pressure dependence of elastic constants and the density of solids has been applied to polycrystalline KCl to pressures of $\mathbf{3 6}$ kilobars. Simultaneous measurement of longitudinal and transverse wave velocities in a specimen of initial thickness of approximately $0.2 \mathrm{~mm}$, compressed between two tungsten carbide anvils, yields the adiabatic pressure derivative of density, which is numerically integrated to give the pressure-density relation, permitting direct comparison with Bridgman's data. Densities obtained by the present method are within 0.7 per cent of Bridgman's throughout the pressure range studied. The $19.7-\mathrm{kb}$ phase transition of $\mathrm{KCl}$ is marked by a 6 and 12 per cent increase in bulk and shear moduli, the former in good agreement with Bridgman. Extension of the present method to higher pressures and high temperatures and to a variety of materials appears feasible.
\end{abstract}

Introduction. The pressure dependence of elastic constants of selected solids has been measured by uitrasonic pulse travel time [Lazarus, 1949; Hughes, 1960] and by ultrasonic interference methods [McSkimin, 1958] to pressures of $10 \mathrm{~kb}$. The pressure-volume measurements of Bridgman give densities and compressibilities to pressures of $100 \mathrm{~kb}$ under hydrostatic conditions.

In the present experiment the ultrasonic pulse interference methods developed by McSkimin [1950] are used in a pressure cell of the type first developed by Bridgman [1952] and later modified by Griggs and Kennedy [1956] to determine the variation of the elastic constants and the density of a polycrystalline material, $\mathrm{KCl}$ with pressure. This material was chosen because the dependence of the elastic constants and of the density of the low-pressure phase on pressure had been well established [Lazarus, 1949], and because $\mathrm{KCl}$ is known to have a polymorphic phase transition [Bridgman, 1940] at a pressure readily attainable in our present pressure cell. The present experimental technique, while similar to that of Anderson [1960], should permit extension of pressure-dependence studies of elastic constants and density of solids to the limits of the Bridgman-type anvil apparatus. Harris, Vaisnys, Stromberg, and Jura,

\footnotetext{
1 In partial fulfillment of the requirements for the degree of Doctor of Philosophy.

2 Contribution 10.
}

[1960] report achieving pressures of $400 \mathrm{~kb}$ in such an apparatus. Straightforward modifications of the present equipment should permit the introduction of temperature as a variable in the measurements.

Description and theory of measurement. $M c$ Skimin [1950] developed a method for obtaining elastic constants of small specimens by measuring the carrier frequency of an ultrasonic RF pulse reflected from opposite sides of the specimen. If the pulse duration is long in comparison with the transit time, the carrier frequency may be varied until a condition of destructive interference between pulses reflected at opposite sides of the specimen is produced. From specimen thickness and carrier frequency it is then possible, after some corrections, to determine elastic wave velocities to high precision. A buffer rod is often useful in separating the quartz transducer from the specimen, especially when the conditions of stress or temperature imposed upon the specimen are unfavorable for the operation of the quartz transducer [McSkimin, 1953].

In the present experiment (Figs. 1 and 2) an axial force is applied through two tungsten carbide inserts to a thin specimen, of the order of $0.2 \mathrm{~mm}$ thick and contained laterally by a pipestone washer. At the stress-free sides of the inserts are two quartz transducers, $x$-cut and $y$ cut. An RF pulse is applied to either transducer. The resulting ultrasonic pulse is internally re- 


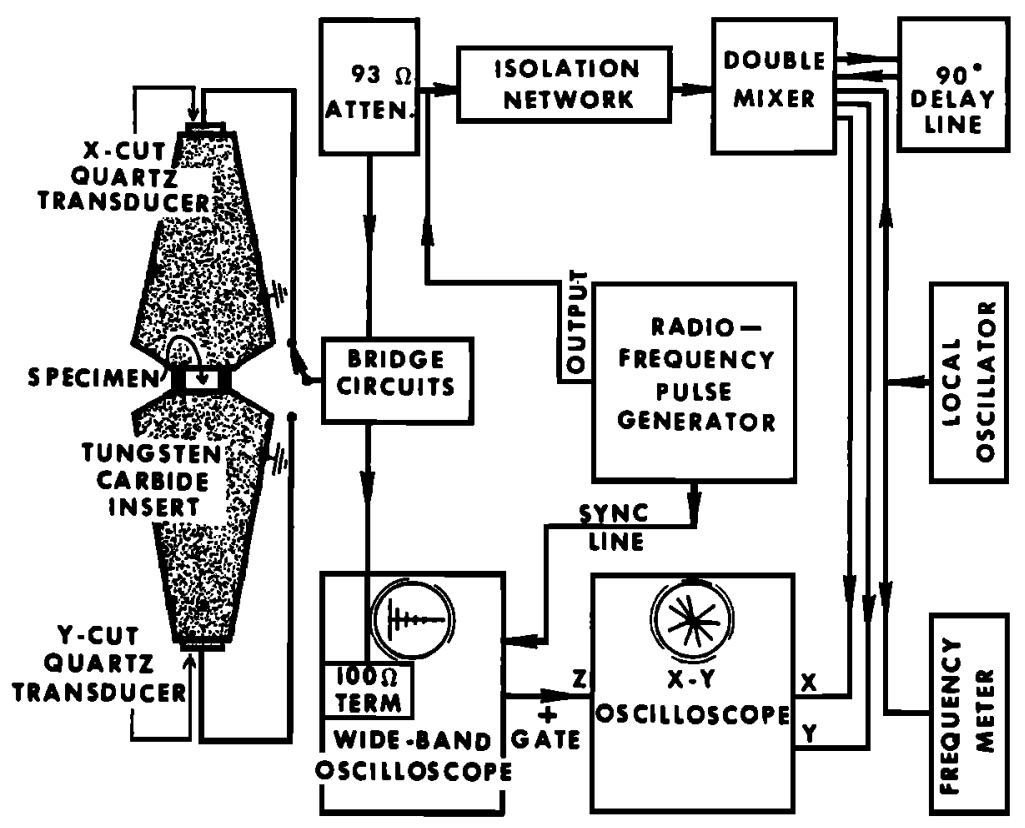

Fig. 1. Block diagram of high-pressure ultrasonic interferometer.

flected within the insert, as many as ten successive reflections being observed. Each reflection is detected by the transmitting crystal and displayed on the wide-band oscilloscope. In most applications, including the present, the acoustic impedance of the tungsten carbide exceeds that of the specimen. Therefore, when the wavelength in the specimen is an integral multiple of twice the thickness, the multiple reflections transmitted from within the sample will all be in phase with each other and $180^{\circ}$ out of phase with the reflection from the carbidespecimen interface. The reflected pulse amplitude will be zero when the carrier frequency is

$$
F_{n}=n v / 2 l
$$

where $v$ is the elastic wave velocity (longitudinal or transverse) in the sample, $l$ the sample thickness, $F_{n}$ the frequency of the $n$th minimum, and $n$ an integer (Appendix). If the frequency of two adjacent minima can be measured, $n$ is uniquely determined by

$$
n=F_{n} / f
$$

where $f=F_{n+1}-F_{n}$. Since no bonding materials are used between anvil and specimen, no correc- tions are required for bond phase shifts. High pressure and clean surfaces insure adequate acoustic coupling.

To determine the variation of elastic constants and density with pressure, changes in elastic wave velocities as well as in physical dimensions of the specimen must be taken into account. Cook [1957] has given an analytical procedure for determining the variation of density with pressure for a specimen under hydrostatic compression, from the measured frequencies required for destructive interference of longitudinal and transverse wave pulses and from the initial specimen thickness.

Following Cook and with the assumption of hydrostatic stress distribution in the specimen, to be verified in the present experiment by comparison with Bridgman's data obtained under isothermal conditions, we write the adiabatic bulk modulus as

$$
k_{\mathrm{ad}}=\rho(d P / d \rho)_{\mathrm{ad}}=\rho\left(v_{p}{ }^{2}-4 v_{a}^{2} / 3\right)
$$

where $\rho, v_{p}$, and $v_{s}$ are the density, the longitudinal, and the transverse wave velocities, respectively. Since the static compression is nearly isothermal, a small correction to convert the adiabatic to the isothermal bulk modulus is re- 


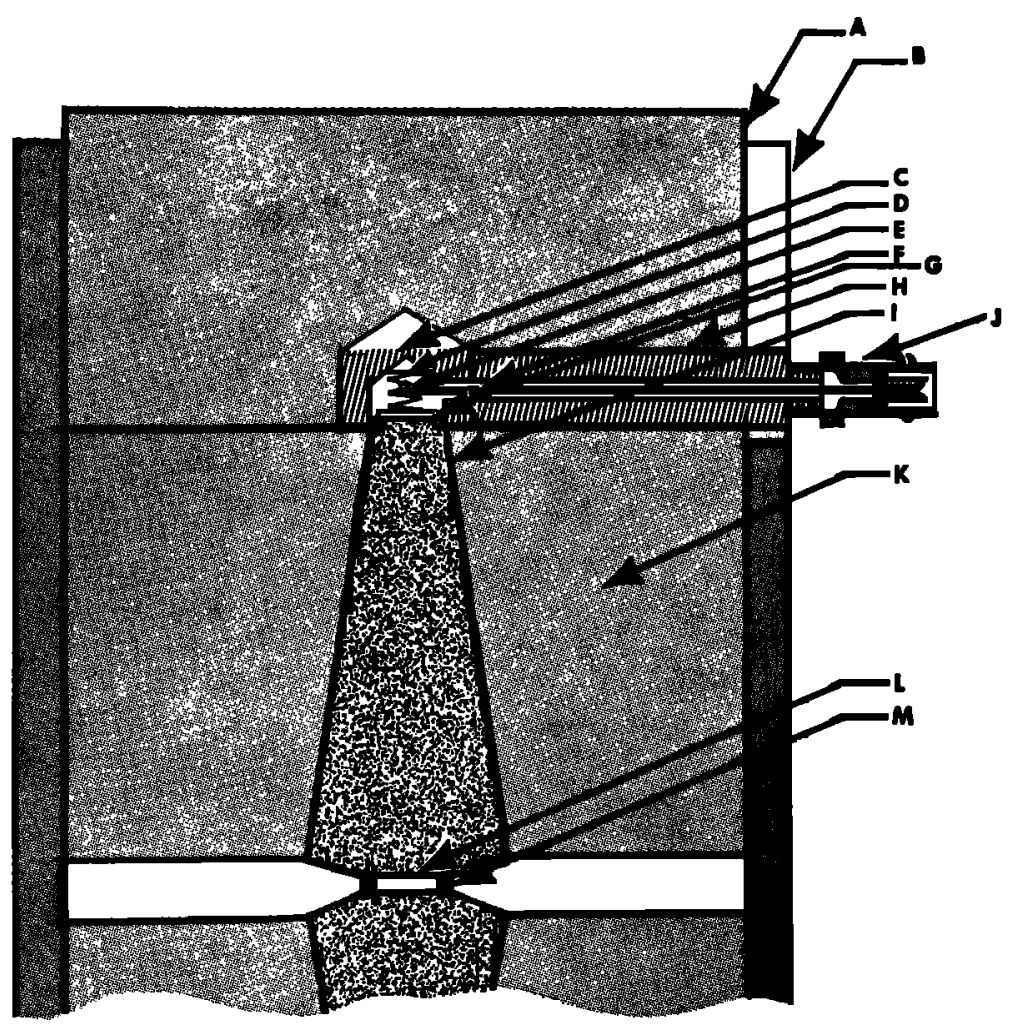

Fig. 2. Pressure cell assembly, upper part (lower part is identical to upper). $A$, piston cap (alloy steel); $B$, alignment tube (mild steel); $C$, insulating cap (lucite); $D$, contact spring (steel) ; $E$, soldered connection; $F$, center conductor (11-gage copper) ; $G$, quartz transducer; $H$, insulating sleeve (lucite); $I$, tungsten carbide insert wrapped in 0.001 -inch lead foil; $J, \mathrm{BNC}$ connector; $K$, piston (alloy steel); $L$, specimen; $M$, pipestone washer.

quired. For polycrystalline $\mathrm{KCl}$ the ratio of these bulk moduli is ${ }^{3}$

$$
k_{\mathrm{ad}} / k_{\mathrm{ieo}}=1+\Delta=1.040
$$

Initial specimen thickness, $l_{0}$, and thickness $l$ at pressure $P$ are related by

$$
l=\dot{i_{0}} / s
$$

which leads, under the assumption of hydrostatic stress distribution, to

$$
\rho=\rho_{0} s^{8}
$$

where $\rho_{0}$ is the initial density, $s$ is to be determined experimentally, and all quantities are taken under isothermal conditions. If the dif-

\footnotetext{
' Obtained from $\Delta=9 \alpha^{2} T k_{\mathrm{ad}} / \rho_{0} c_{p}$, where $\alpha$ is the linear thermal expansion coefficient, $T$ the temperature, and $c_{p}$ the specific heat at constant pressure.
}

ference in the frequencies of successive first reflection minima is denoted by $f_{p}$ for longitudinal and by $f$, for transverse waves, substitution of $(1),(2),(4),(5)$, and (6) into (3) yields (cf. Cook)

$$
s=1+\frac{1+\Delta}{12 l_{0}^{2} \rho_{0}} \int_{0}^{P} \frac{d P}{f_{p}^{2}-4 f_{s}^{2} / 3}
$$

Measurement of $l_{0}$, required in (7), is made difficult by the fact that initially the specimen is uneven, soft, and thin. It must be several thousandths of an inch thinner than the washer to prevent extrusion and smaller in diameter to permit proper mounting. As the pressure is increased, the pipestone washer expands radially inward until it meets the specimen. Thickness $l_{0}$ was calculated from specimen mass $m$, crystal density $\rho_{0}$, and diameter $d$ by assuming that, when good acoustic coupling is achieved, the specimen diameter is equal to the initial speci- 


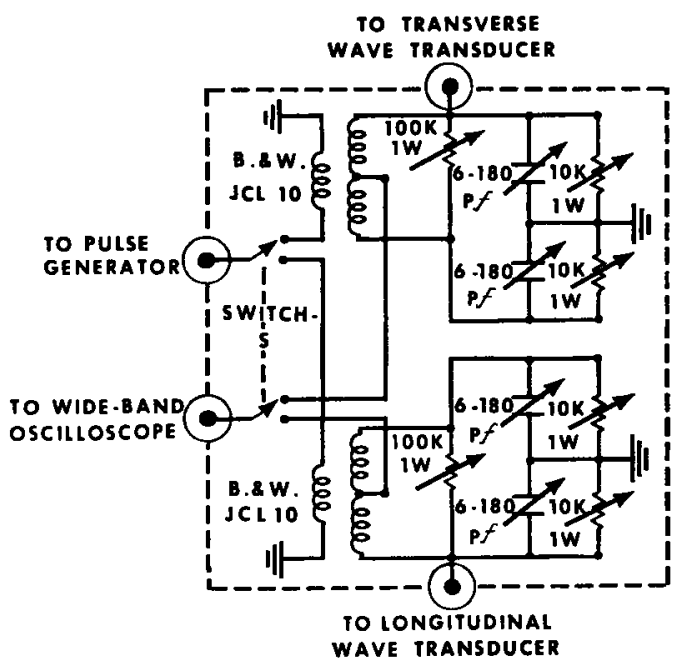

Fig. 3. Bridge circuits (after Arenberg): two bridge circuits allow optimum adjustment for each transducer.

men diameter. With these quantities (7) may be written

$s=1+\frac{(1+\Delta) \pi^{2} d^{4} \rho_{0}}{192 m^{2}} \int_{0}^{P} \frac{d P}{f_{\nu}^{2}-4 f_{s}^{2} / 3}$

Density is obtained from (6) and wave velocities from (5) and (1). The adiabatic bulk and rigidity moduli $k_{\mathrm{ad}}$ and $\mu$ then follow from (3) and

$$
\mu=\rho v_{s}^{2}
$$

Poisson's ratio is given by

$$
\sigma=0.5\left(k_{\mathrm{ad}}-2 \mu / 3\right) /\left(k_{\mathrm{ad}}+\mu / 3\right)
$$

The pressure derivative of density, measured in the present method, must be integrated as in (8) for comparison with Bridgman's data. This numerical integration is valid as long as there are no first-order discontinuities in the $\rho$ versus $P$ relationship. At a polymorphic transition, such as that in $\mathrm{KCl}$, another approach must be devised. For a material for which Bridgman has measured the density increase, the density of the high-pressure phase may be used to start the integration procedure above the transition pressure. From the resulting $\rho$ versus $P$ data the values of the elastic constants follow as before. As is evident from (1) to (6), the integrand of (7) would become infinite at the transition pressure. However, if the transition were spread out over a range of pressures, the variation with pressure of the integrand of (7) would be a bell-shaped curve, the width of which would depend upon the range of pressures over which the transition occurs. The known density change [Bridgman, 1940] at the transition was taken into account by finding

$$
\Delta S=\left(\rho_{B}{ }^{1 / 3}-\rho_{A}{ }^{1 / 3}\right) \rho_{0}{ }^{-1 / 3}
$$

where $\rho_{A}$ and $\rho_{B}$ are the densities below and above the transition, respectively. For substances with sluggish transitions the validity of performing the integration in (8) directly remains to be investigated.

High-pressure system. The pressure cell (Fig. 2) consists of two tungsten carbide inserts, ${ }^{4}$ each forced into a piston of hardened alloy steel. 5 The pistons are free to slide in a tube which provides the necessary alignment. Lead foil, 0.001 inch thick, wrapped around the inserts, prevents tangential loading of the carbide. The load-bearing and stress-free faces of the inserts

${ }^{4}$ Firth Sterling H-6 (94\% WC, 6\% Co) obtained from Carbide Specialty, Inc., McKeesport, Pa.

${ }^{5}$ Vascojet 1000, gift from Vanadium-Alloys Steel Corp., Latrobe, Pa., hardened to $\mathbf{R}_{c} 50$.

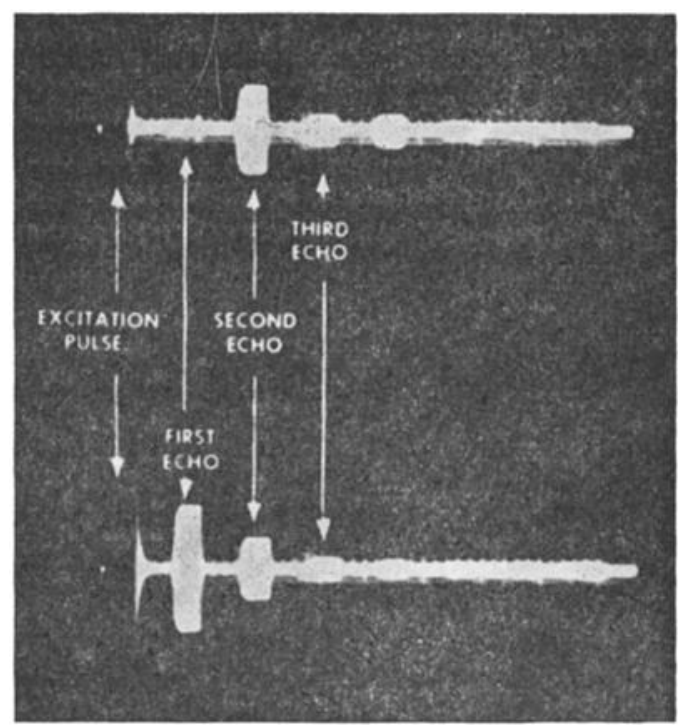

Fig. 4. Ultrasonic reflections (10- $\mu$ sec duration) within carbide inserts on wide-band oscilloscope; upper, minimum in first reflection from carbidespecimen interface; lower, reflections away from minimum frequency. 


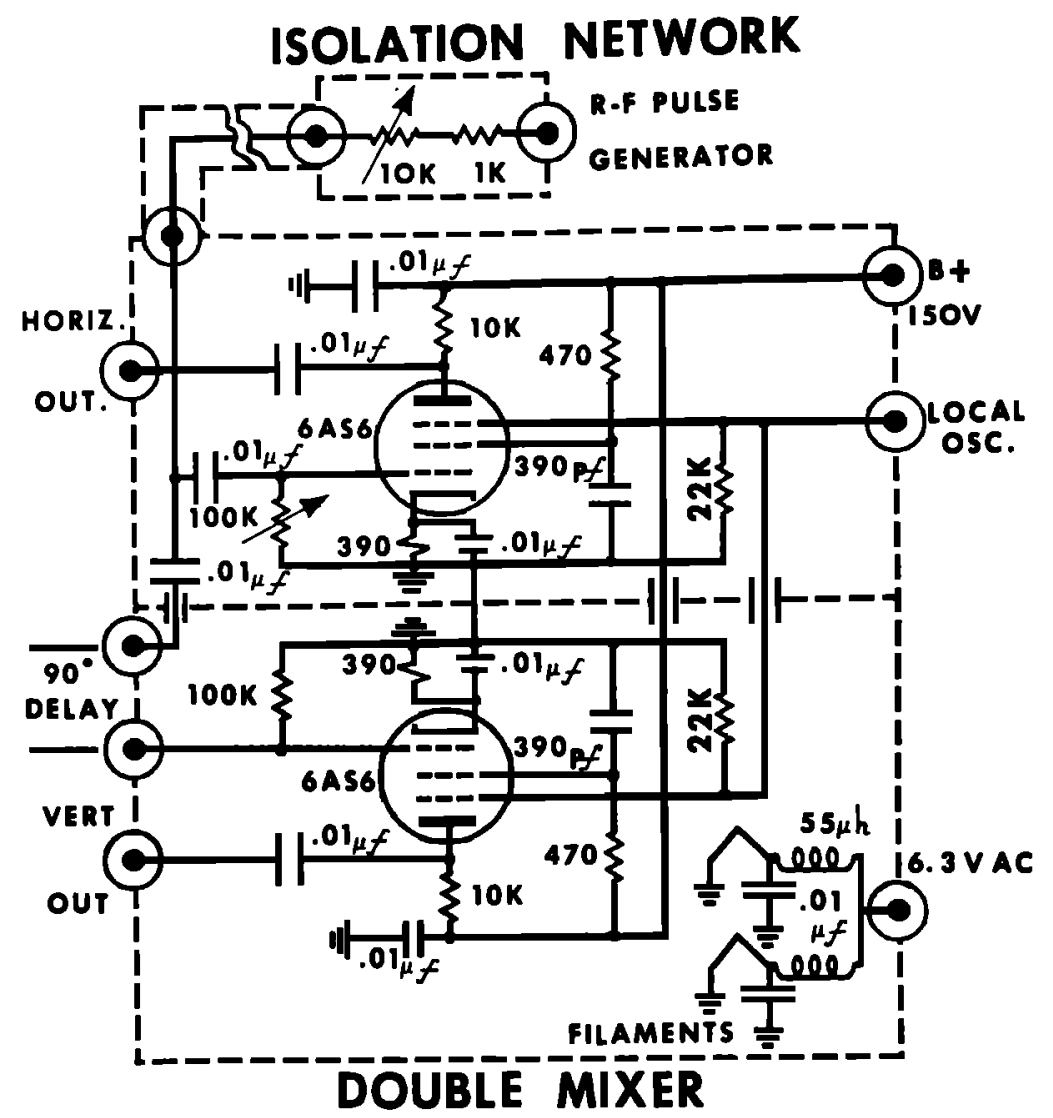

Fig. 5. Isolation network and double mixer (after McSkimin).

are ground parallel to 0.00003 inch. The overall dimension of the cell is 11 inches.

The force on the pressure cell is applied by means of a 100 -ton hydraulic press, which produces a nominal maximum pressure of $70 \mathrm{~kb}$ on the 0.5-inch-diameter bearing face of the carbide inserts. Pressure is calculated from the area of the carbide inserts and the force exerted on the pressure cell, obtained from the ram area and the oil pressure. A Heise gage with a 12-inchdiameter scale and full scale deflection of 5000 $\mathrm{lb} / \mathrm{in}$ " measures oil pressure with a precision of $5 \mathrm{lb} / \mathrm{in}$ ".

Ultrasonic system. An RF pulse generator ${ }^{\theta}$ (Fig. 1) provides the excitation to either a 40$\mathrm{Mc} / \mathrm{s} x$-cut or a $30-\mathrm{Mc} / \mathrm{s} y$-cut quartz transducer. The transducers are bonded to the stressfree surfaces of the carbide inserts with phenyl-

- Model G-65OC, Arenberg Ultrasonic Laboratory, Jamaica Plain, Mass. salicylate. The RF pulse is applied through a 93-ohm attenuator, switch $S$, and a step-up transformer, to one of two bridges (Fig. 3). One bridge arm is connected to the transducer. With the bridge in balance, the bridge output observed on the oscilloscope is zero during the application of the excitation pulse. On the other hand, since the signal produced by reflections is applied across one arm of the bridge only, it produces a differential voltage which is readily detected without overdriving the oscilloscope amplifier, although more than $80 \mathrm{db}$ down from the excitation pulse. The RF pulse may be applied to either the $x$-cut or $y$-cut transducer by means of switch $S$, permitting convenient and quick change in viewing longitudinal or transverse wave reflections.

As the carrier frequency is tuned (Fig. 4), the first reflection goes through a null (upper ex-

7 Tektronix type 543 with type L preamplifier. 


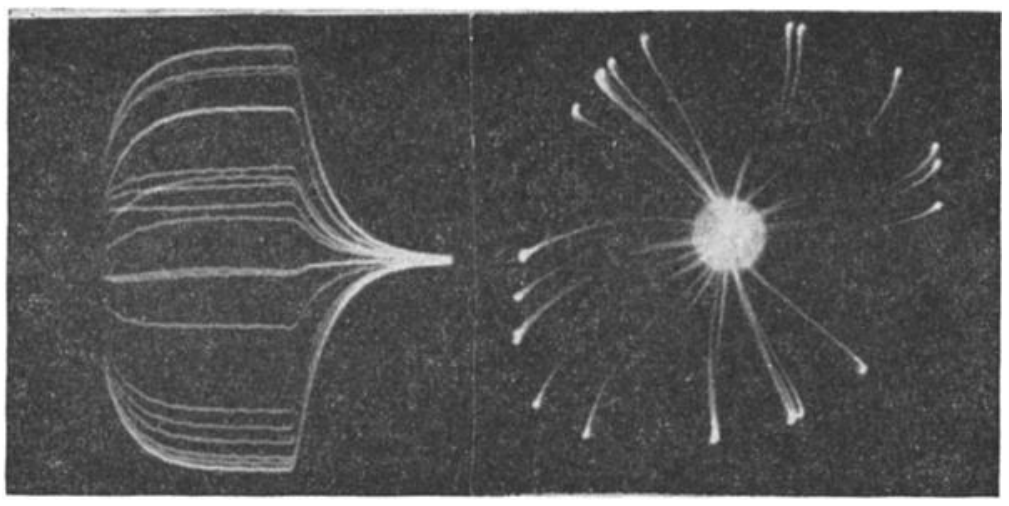

Fig. 6. Traces on $x-y$ oscilloscope when pulse carrier and local oscillator frequencies are equal; left, oscilloscope operating with driven sweep and output from half of double mixer applied to vertical amplifier; right, oscilloscope operating in $x-y$ mode.

posure). The frequencies of minima of successive reflections differ by approximately $50 \mathrm{kc} / \mathrm{s}$ owing to the cumulative phase lags introduced by nonparallel surfaces and the transducer-carbide bond. The frequency of the null of the first reflection is affected only by lack of parallelism. The normal appearance of the first four reflections away from the minimum condition appears in the lower exposure.

For the specimen thicknesses used, in the range of $0.2 \mathrm{~mm}$, successive minima for longitudinal waves are approximately $10 \mathrm{Mc} / \mathrm{s}$ apart. In order that these minima may be observed without changing transducers, a considerable bandwidth is required or the transducer must be excited at higher harmonics. May [1954] emphasized the increased bandwidth obtainable with a delay line of very high acoustic impedance. Tungsten carbide, having a very high compressive strength and a density of $15 \mathrm{~g} / \mathrm{cm}^{3}$, is well suited in this case for the dual roles of ultrasonic buffer rod and pressure cell insert. Within the carbide, reflections have been observed from 7 to $51 \mathrm{Mc} / \mathrm{s}$ from the $x$-cut $40-\mathrm{Mc} / \mathrm{s}$ transducer and from 5 to $35 \mathrm{Mc} / \mathrm{s}$ from the $y$-cut $30-\mathrm{Mc} / \mathrm{s}$ transducer. ${ }^{8}$

Frequency-measuring system. The RF pulse generator (Fig. 1) is a pulsed oscillator producing $10-\mu$ sec pulses of $R F$ voltage of variable frequency. The pulse rise and decay times are less than $0.5 \mu \mathrm{sec}$ in the frequency range 5 to 51

\footnotetext{
8 With $v_{p}=6.5 \mathrm{~km} / \mathrm{sec}$ and $v_{s}=4.0 \mathrm{~km} / \mathrm{sec}$ for tungsten carbide, as given by Arenberg [1955], the 3-db bandwidths calculated from formulas of May [1954] are $40 \pm 15 \mathrm{Mc} / \mathrm{s}$ and $30 \pm 11 \mathrm{Mc} / \mathrm{s}$.
}

$\mathrm{Mc} / \mathrm{s}$. To measure the carrier frequency of an RF pulse which at $30 \mathrm{Mc} / \mathrm{s}$ contains only 300 cycles, a more elaborate technique is required than that used for measuring the frequency of a continuous oscillator [Bagley and Hartke]. The RF pulse is applied through a 10,000-ohm potentiometer to the control grid of each 6AS6 tube in the double mixer (Fig. 5). In half of the mixer the RF pulse is heterodyned with the local oscillator voltage, ${ }^{9}$ and the resulting output is applied to the vertical amplifier of the $x-y$ oscilloscope, ${ }^{10}$ operating with a driven sweep. When the carrier and local oscillator frequency are equal, the $\mathrm{RF}$ pulse degenerates into a pulse consisting of a single straight line (Fig. 6, left).

In an alternative and more convenient display, the mixer output is applied to the horizontal amplifier of the $x-y$ oscilloscope. In addition, the RF pulse applied to the other half of the mixer is delayed $90^{\circ}$ by a suitable length of RF cable, ${ }^{11}$ heterodyned with the local oscillator, and applied to the vertical amplifier of the $x-y$ oscilloscope. If successive RF pulses were phase coherent, an inclined straight line would be observed on the oscilloscope when the RF pulse carrier frequency equaled the local oscillator frequency. However, phase incoherence between local oscillator and pulse generator produces a series of spokes on the $x-y$ oscilloscope

- Unit oscillator, type 1211-B, General Radio Co. 10 Dumont oscilloscope, type 248.

11 Three feet of RG-58/U cable provides a 4.5nsec delay. Fine tuning for the $90^{\circ}$ delay is obtained by means of a 50- to 700-pf capacitor in parallel with the RF cable. 


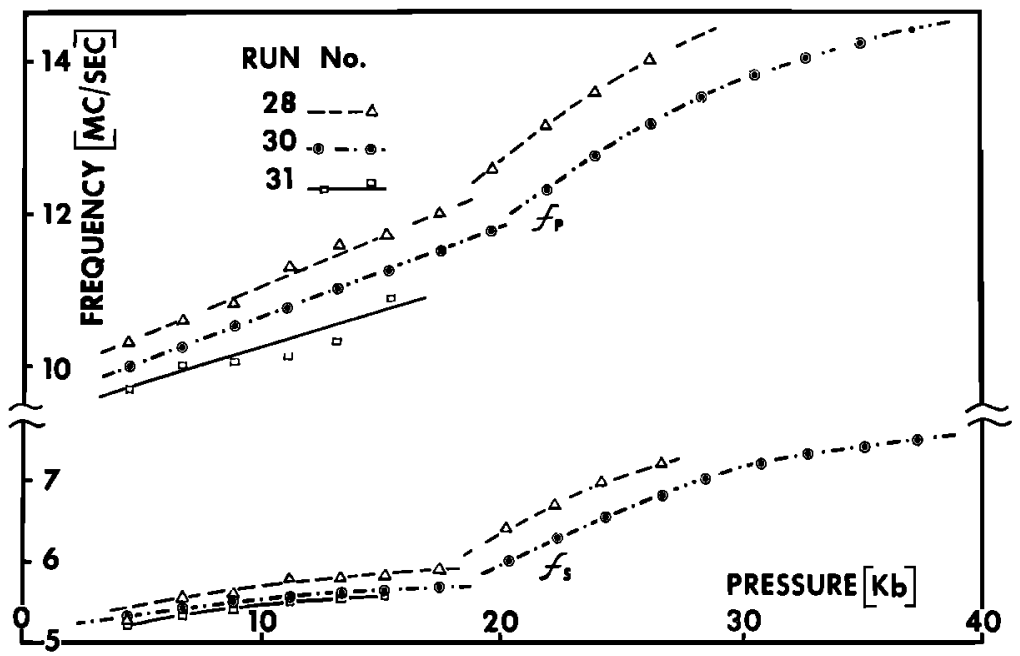

Fig. 7. Frequencies $f_{p}$ and $f_{0}$ versus pressure.

when the local oscillator and carrier frequencies are equal (Fig. 6, right). Because of the low pulse repetition rate, $60 \mathrm{cps}, z$-axis intensification is provided by a synchronized, positive pulse from the wide-band oscilloscope. The local oscillator frequency is monitored by a frequency meter, ${ }^{12}$ calibrated against radio station WWV. Frequency measurements can be repeated to 0.005 per cent.

Experimental procedures and calculations. The specimens are prepared by compaction of powdered $\mathrm{KCl}$ into disks 0.0075 inch thick and 0.400 inch in diameter in a die with a force of 40 tons. Flat surfaces are obtained by lapping on a damp nylon cloth. Pipestone washers, internal diameter of $0.40 \mathrm{inch}$, external diameter of $0.50 \mathrm{inch}$, and thickness of $0.015 \mathrm{inch}$, are used to prevent extrusion of specimens under pressure. The washers, precoated with jeweler's rouge, help to achieve an approximately hydrostatic environment in the specimen while it is being squeezed between the bearing surfaces of the inserts. The washers are effective in preventing extrusion only when the specimen is several thousandths of an inch thinner than the washers.

Pressures of $30 \mathrm{~kb}$ on the specimen-washer combination apparently cause plastic dishing deformation [Griggs and Kennedy, 1956; Balchan and Drickamer, 1961] of the order of 0.0001

12 Berkeley model 5570 frequency meter. inch. As a result, interferometry on the next pressure application is difficult unless the workhardened surfaces are reground. The dishing that occurs while the specimen is under pressure does not appear to have an adverse effect upon the measurements if the inserts are initially flat. The transducers are 0.375 inch in diameter, so that the beamwidth is limited to the central high-pressure region in the specimen. The data consist of frequencies $F_{n}$ required for destructive intereference in the $n$th mode as a function of pressure. For the three specimens, values of $F_{n}$ for longitudinal waves are in the neighborhood of $10 \mathrm{Mc} / \mathrm{s}$ for $n=1,20 \mathrm{Mc} / \mathrm{s}$ for $n=2$, and $30 \mathrm{Mc} / \mathrm{s}$ for $n=3$. Corresponding values for transverse waves are 5,10 , and 15 $\mathrm{Mc} / \mathrm{s}$. The measured values of $F_{n}$ and (2) are used to plot $f_{p}$ and $f_{0}$ (Fig. 7). The three curves should not coincide because each depends on the initial thickness, which was different for each specimen. For $\mathrm{KCl}$ the values of $f_{p}$ and $f_{0}$ are independent of $n$ within the experimental error of frequency measurement. However, preliminary results for bismuth suggest some dispersion. The integrand in (8) is calculated and plotted, and an average curve for the data from the three specimens is drawn. Integration then yields $s$. The scatter in the integrand for the three specimens is such that after integration the density is determined to better than \pm 0.3 per cent. Density and thickness are obtained from (8), (6), and (5). Equations 5, 2, and 1 yield 


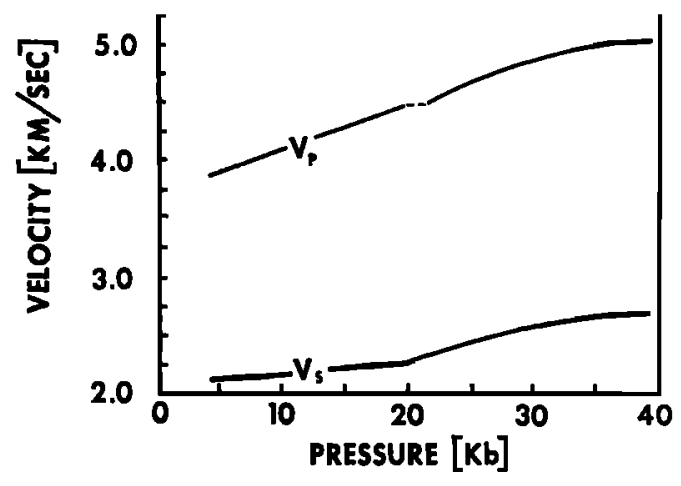

Fig. 8. Calculated longitudinal and transverse velocities versus pressure.

wave velocities (Fig. 8). Bulk and rigidity moduli and Poisson's ratio (Fig. 9) are obtained from (3), (9), and (10). Experimental errors introduced in selecting the minimum and in measuring frequency ( \pm 1 per cent) and initial thickness $( \pm 0.8$ per cent) result in estimated maximum errors of \pm 0.3 per cent in density as given above, \pm 2 per cent in wave velocities, and \pm 4 per cent in elastic constants. The largest error is introduced by the measurement of initial thickness. While this affects the absolute values of density, velocities, and elastic constants, it has no effect on their relative values. Four determinations of the transition pressure (Fig. 7) give $18.8 \pm 1.5 \mathrm{~kb}$, as compared with the 19.7-kb transition found by Bridgman [1940] with a density change $\Delta \rho=$ $0.1133 p_{0}$. This is used in (11) and (8) to calculate $s$ above the transition. Density, thickness, velocities, and elastic constants then follow directly by the same procedure as before.

Conclusion. In the present method for measuring density and elastic constants of solids as a function of pressure a thin disk-shaped specimen is placed under axial compression and confined laterally by an annular pipestone washer, in the familiar geometry first developed by Bridgman. An ultrasonic RF pulse, produced by quartz transducers, which are mounted outside the stress field, is transmitted through a tungsten carbide anvil into the specimen. The contrast in acoustic impedances between anvil and specimen produces a $180^{\circ}$ phase shift between the signals reflected at the two anvil-specimen interfaces. This results in destructive interference for ultrasonic RF pulses, the carrier fre- quency of which is such that the specimen thickness is an integral number of half-wavelengths, provided that the pulse duration is long in comparison with specimen transit time. Measurement of these resonant frequencies for both longitudinal and transverse waves as a function of pressure and of the initial dimensions of the specimen permits calculation of the pressure derivative of the density, which may be integrated directly to give both the density and the thickness of the specimen. From these the elastic wave velocities and constants are obtained.

The application of this method is straightforward as long as the density-pressure relation is continuous. When a discontinuity produced by a polymorphic transition exists, the method may be used below the transition. If the density jump is known independently, the method may also be applied above the transition, yielding density, velocities, and elastic constants as functions of pressure. For some materials with sluggish transitions, preliminary results suggest that this method may give valid results without independent information on the density jump. The results for density of the first material studied, polycrystalline $\mathrm{KCl}$, are in close agreement with Bridgman's results, being within 0.7 per cent of his values at all pressures. The increase in bulk modulus of 6 per cent at the 19.7-kb transition compares with 8 per cent obtained from differentiating Bridgman's data. The rigidity modulus increases 12 per cent. The close agreement in the measurement of volumes between hydrostatic pressure methods and the present results using axial compression, suggests that the stress distribution in the latter must be nearly hydrostatic or that shear stresses have only a slight effect on wave velocities.

Because of its lattice structure and its low shear strength, the choice of $\mathrm{KCl}$ was particularly favorable for developing nearly hydrostatic conditions.

The present method should be applicable to a variety of solids and at much greater pressures. The present limitation appears to be the plastic dishing deformation near the edges of the tungsten carbide anvils, which reduces the sharpness and affects the frequency of the minimum. The use of smaller-diameter quartz transducers would reduce the beamwidth of the ultrasonic pulse and should permit a considerable increase 


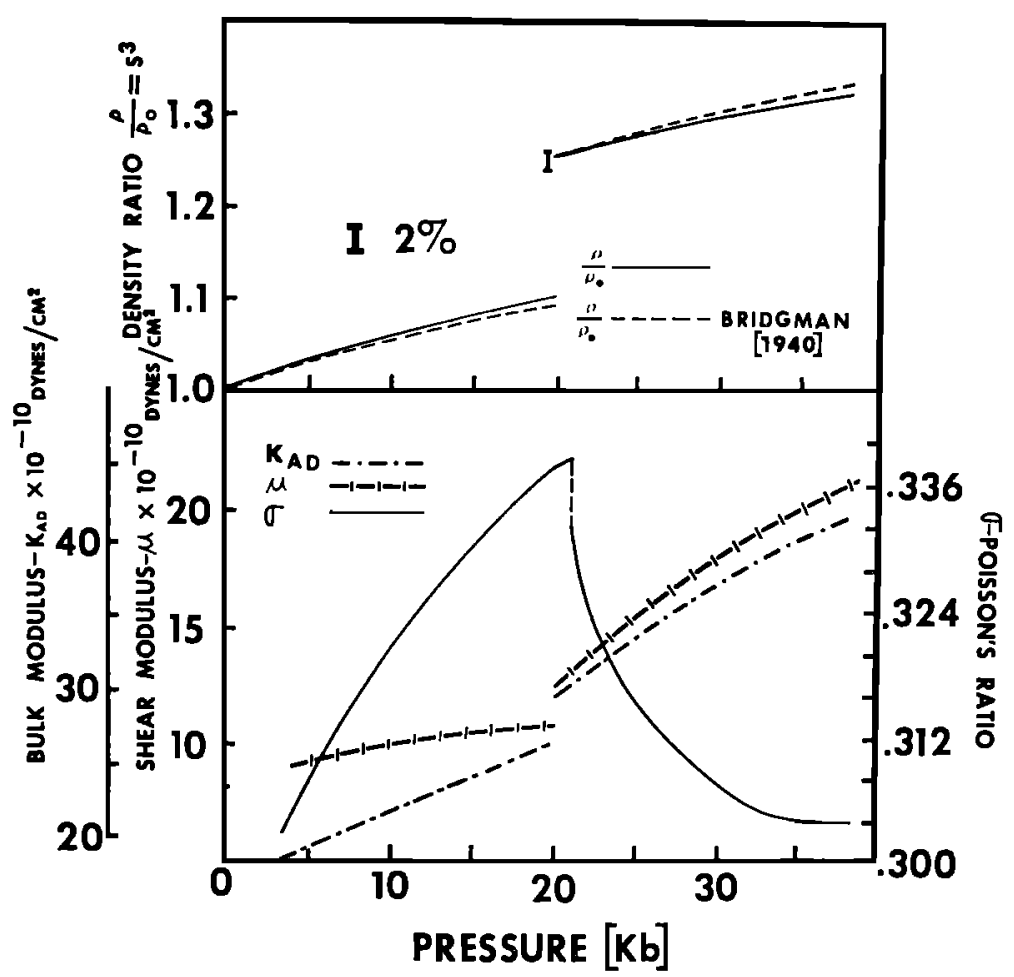

Fig. 9. Mean density, adiabatic bulk modulus, rigidity, and Poisson's ratio versus pressure.

in the pressure range. Measurement over a wide temperature range also appears feasible.

\section{APPENDIX}

Rayleigh [1960] has analyzed the reflection and transmission of sound of frequency $F$ from a layer of thickness $L$, sound velocity $V_{1}$, density $\rho_{1}$, lying between two identical, semi-infinite media with sound velocity $V$ and density $\rho$. At normal incidence, the reflection coefficient is

$$
\begin{aligned}
&\left(\frac{\rho V}{\rho_{1} V_{1}}-\frac{\rho_{1} V_{1}}{\rho V}\right) {\left[4 \cot ^{2}\left(\frac{2 \pi L F}{V_{1}}\right)\right.} \\
&\left.+\frac{\rho V}{\rho_{1} V_{1}}+\frac{\rho_{1} V_{1}}{\rho V}\right]^{-1 / 2}
\end{aligned}
$$

which vanishes for $F=n V_{1} / 2 L$, in agreement with the destructive interference condition given by (1). From (12) and the appropriate constants for longitudinal waves for $\mathrm{KCl}$ and $\mathrm{WC}$, given above, an estimate for sharpness of tuning at the minimum, as measured at the onetenth maximum amplitude points is $\pm 147 \mathrm{kc} / \mathrm{s}$.
This is in qualitative agreement with the observed value.

Acknowledgments. D. L. Arenberg, H. J. McSkimin, and D. G. Gisser, provided assistance in the design of the electronic circuitry. T. E. Davidson helped in the design of the pressure cell which was constructed by C. T. Premo. G. Lehmberg assisted in gathering data and performing calculations. The opportunity extended by Professor Francis Birch of carrying out some of the initial experiments at the Dunbar Laboratory, Harvard University, is gratefully acknowledged. This research was supported in part by the National Science Foundation under grants G-5240 and G9374, the National Aeronauties and Space Administration under grant NsG-100-60, and the Research Grants Committee of Rensselaer Polytechnic Institute.

\section{REFERENCES}

Anderson, O. L., The accurate determination of the equation of state by ultrasonic measurements, Progress in Very High Pressure Research, John Wiley \& Sons, New York, 314 pp., 1960.

Arenberg, D. L., Final Report, Contract NONR $794(00), 1955$. 
Bagley, A., and D. Hartke, Measurement of the carrier frequency of $\mathrm{r}-\mathrm{f}$ pulses, Hewlett Packard Application Note 3 (undated).

Balchan, A. S., and H. G. Drickamer, High pressure resistance cell, and calibration points above 100 kilobars, Rev. Sci. Instr., 32, 308-313, 1961.

Bridgman, P. W., The compression of 46 substances to $50,000 \mathrm{~kg} / \mathrm{cm}^{2}$, Proc. Am. Acad. Arts Sci., 74, 21-51, 1940.

Bridgman, P. W., The resistance of 72 elements, alloys and compounds to $100,000 \mathrm{~kg} / \mathrm{cm}^{2}$, Proc. Am. Acad. Arts Sci., 81, 165-251, 1952.

Cook, R. K., Variation of elastic and static strains with hydrostatic pressure, A method for calculation from ultrasonic measurements, $J$. Acoust. Soc. Am., 29, 445-449, 1957.

Griggs, D. T., and G. C. Kennedy, A simple apparatus for high pressures and temperatures, Am. J. Sci., 254, 722-735. 1956.

Harris, R. E., R. J. Vaisnys, H. Stromberg, and G. Jura, Resistance and thermal gap measurements to 400,000 atmospheres, Progress in Very High Pressure Research, John Wiley \& Sons, Inc., New York, 314 pp., 1960.

Hughes, D. S., Properties of rocks under high pressure and temperature, Methods and Techniques in Geophysics, 1, Interscience Publishers, New York, 374 pp., 1960.

Lazarus, D., The variation of the adiabatic elastic constants of $\mathrm{KCl}, \mathrm{NaCl}, \mathrm{CuZn}, \mathrm{Cu}$ and $\mathrm{Al}$ with pressure to 10,000 bars, Phys. Rev., 76, 545-553, 1949.

May, J. E., Characteristics of ultrasonic delay lines using quartz and barium titanate ceramic transducers, J. Acoust. Soc. Am., 26, 347-353, 1954.

McSkimin, H. J., Ultrasonic measurement techniques applicable to small solid specimens, $J$. Acoust. Soc. Am., 22, 413-418, 1950.

McSkimin, H. J., Measurement of elastic contants at low temperatures by means of ultrasonic waves-data for silica and germanium single crystals, and for fused silica, J. Appl. Phys., 24, 988-997, 1953.

McSkimin, H. J., The elastic moduli of single crystal germanium as a function of hydrostatic pressure, J. Acoust. Soc. Am., 30, 314-318, 1958.

Rayleigh, John William Strutt, 3rd baron, The Theory of Sound, II, p. 88, Dover Publications, New York, 504 pp., 1960.

(Manuseript received March 3, 1962; revised April 10, 1962.) 